\title{
Workshop on Extreme Physics
}

\section{Organizers: Carole Mundell ${ }^{1}$ \& Mark Sullivan ${ }^{2}$}

\author{
${ }^{1}$ Astrophysics Research Institute, Liverpool John Moores Univ., Birkenhead, CH41 1LD, UK \\ email: cgm@astro.livjm.ac.uk \\ ${ }^{2}$ Department of Physics, University of Oxford, Oxford, OX1 3RH, UK
}

Summary. Never before has there been such a wealth of versatile ground- and space-based facilities with which to detect variable emission across the electromagnetic spectrum and beyond, to non-EM signals such as neutrinos and gravitational waves, to probe the most extreme phenomena in the Universe. The variable sky is already providing a wealth of new and surprising observations of phenomena such as GRBs, SNe and AGN that are pushing current theories beyond the state of the art. Multi-messenger follow-up will soon become de rigeur, and upcoming radio and optical all-sky transient surveys will revolutionise the study of the transient Universe. In addition to the technical and data challenges presented by such surveys, a major new challenge will be the interpretation of the wealth of available data and the identification of the underlying physics of new classes of variable (and potentially exotic) objects. Theoretical predictions will be vital for interpreting these future transient discoveries.

The goal of this workshop was to bring together theorists and observers in order to identify unexplored synergies across three main research areas of extreme physics: gamma-ray bursts, supernovæ and, more generically, relativistic jets. It aimed to discuss key outstanding questions in these rapidly moving fields, such as the composition and acceleration of GRB and AGN jets, GRB progenitors and central engines, the origin of the wide range of observed variability time-scales in GRB prompt and after-glow light curves and related cosmological applications, the physics of the newly-discovered ultra-luminous SN-like optical transients - as well as to speculate on what we might hope to discover with future technology.

The workshop absorbed two 90-minute sessions, selecting 3 main science topics (Relativistic Jets, GRBs and SNe) which it organised as structured discussions driven by a series of short but provocative questions. The final session featured a panel-led debate but with full audience participation. 